\title{
Intelligent Control Agent for Transient to an Island Grid
}

\author{
J. Rocabert ${ }^{1}$, Student Member, IEEE, G. Azevedo ${ }^{2}$, Student Member, IEEE, G. Vazquez ${ }^{1}$, Student Member, IEEE, \\ I. Candela ${ }^{1}$, Member, IEEE, P. Rodriguez ${ }^{1}$, Member, IEEE, J. M. Guerrero ${ }^{3}$, Senior Member, IEEE. \\ ${ }^{1}$ Electrical Engineering Department \\ Technical University of Catalonia \\ (UPC) Terrassa, Spain \\ Contac Author: \\ rocabert@ee.upc.edu \\ ${ }^{2}$ Department of Electrical \\ Engineering \\ Federal University of Pernambuco \\ (UFPE) \\ Recife, Pernambuco Brazil \\ ${ }^{3}$ ESAII Department \\ Technical University of Catalonia \\ (UPC) \\ Barcelona, Spain
}

\begin{abstract}
The increasing penetration of generation power plants based on renewable energies in the electrical networks has boosted the number of systems connected to the grid. In this scenario the microgrid, providing advanced functionalities to improve the stability and operation of the network, have become very popular. In this paper a control strategy for the microgrid management is presented. The proposed system improves the performance of microgrids and its interaction with the main network, or another microgrid, under grid voltage transients. This technique gives rise to a simple and robust control scheme to ensure the appropriate microgrid disconnection from the main grid. In the following the control algorithm for the intelligent connection agent will be shown, and the grid synchronization system, as well as the voltage and current control loops will be detailed. Finally simulation results obtained using the MATLAB/Simulink \& Psim platform will be presented and discussed
\end{abstract}

\section{INTRODUCTION}

The increasing penetration of renewable energy sources is giving rise to a challenging scenario in the electrical power systems, where the integration of such energy sources must be combined with an ever increasing demand for electrical energy.

Classical power systems structure is characterized by large energy generators and loads connected by radial transmission systems. Over the last years, the great increase of renewable energy sources, among others, has contributed to change this structure that is moving towards a distributed power generation system, where meshed networks connect multiple low scale producers and consumers.

The integration of Distributed Generation is increasing significantly in the last years, mainly, due to the integration of photovoltaic and wind power plant in medium and low voltage grids. Lately, storage energy systems are being integrated into the electrical grid with the aim of improving stability and quality service. In this respect, the short and long term energy storage units can play a major role in the control and operation of microgrids.
The growth of renewable energy system has launched the connection in parallel of voltage source inverters in small isolated grids of photovoltaic generation, batteries and fuel cells, for example. Actually, the same VSI inverters are used to control of weak networks.

In island grids, is usual the use of renewable sources and other small scale distributed generators equipped with voltage source inverter. Similarly, in Uninterruptible Power Supplies (UPS) applications, parallel redundant inverters are used to increase the reliability of the electrical network. In a near future, a lot of distributed generators with dc-ac converters will feed the utility networks, so a good performance of those under any electric grid station must be guaranteed.

One of the essential issues in the integration of renewable energies in distributed generation systems is the parallelization of voltage source inverters in islanding applications. This issue was initially tackled in applications related to Uninterruptible Power Supply UPS, where a redundant power source had to feed critical loads

Conventional supervisory control algorithms are focused on achieving an instantaneous shut down of any power source affected by critical contingencies in order to prevent any case of islanding situation [1]-[2]. In the case of wind power systems, this philosophy changed some years ago, being the wind farms required to ride through transient faults and support the grid under such adverse conditions [3].

Operation of grid connected photovoltaic and wind power systems in intentional island mode under grid fault conditions might be used for improving both the network stability and the energy supply. In this way, development and improvement of island mode detection algorithms is a essential issue [4]. Moreover, the conventional operational of microgrids during grid faults will change as well.

There are several references in the literature dealing with connection and disconnection of microgrids from the main electrical network For instance, the implementation of an intelligent and autonomous switch, is presented in [5]. This 
system is based on monitoring the operating conditions of both the microgrid and the main network and, depending on the status of the main network, the microgrid connection or disconnection procedure is triggered. The transition between these two operation modes should be as transparent as possible, from the point of view of the loads, in order to guarantee the maximum possible quality in supplying the loads connected to the microgrid.

In this paper a solution for transient and steady-stage control from grid connected to islanded microgrids will be proposed. In the scope of the paper, a primary control method is presented to achieve a fast transient and a good operability both in grid connected mode and island mode. This strategy presented in the paper is evaluated through simulations.

\section{MicROGRID OPERATION MODE}

In this section the two main operation modes of a microgrid, grid connected and islanded mode, are explained. The intelligent connection agent (ICA) is responsible of switching to the most appropriate operation mode. However, this decision depends mainly on the network voltage conditions.

Fig. 1 shows the utility and the microgrid considered in the purpose of this paper. In this case, the microgrid consists of the ICA and different loads. As shown in Fig.1, the ICA is formed by a VSI, that takes charge of processing the energy delivered/stored by the microgrid, and a controlled switch $\left(S_{i}\right)$, which physically connects the microgrid to the main grid.

\section{A. Grid Connected Mode}

This will be the usual mode, in which the main objective of the intelligent connection agent is to track the grid voltage.

In this mode, all the loads in the microgrid are being fed by the main grid or other power sources linked to the microgrid. Under these operating conditions, in case of having an ICA with a dc bus made by batteries, these would be charged from the grid if necessary.

In the grid connected mode, the control loop acts as a voltage and frequency follower and feeds, or draws, the necessary active and reactive power to collaborate in maintaining the voltage and frequency values within limits set in the grid codes. An example of this application are the socalled grid-supporting inverters, which are designed to flexibly support the voltage and/or frequency of either a

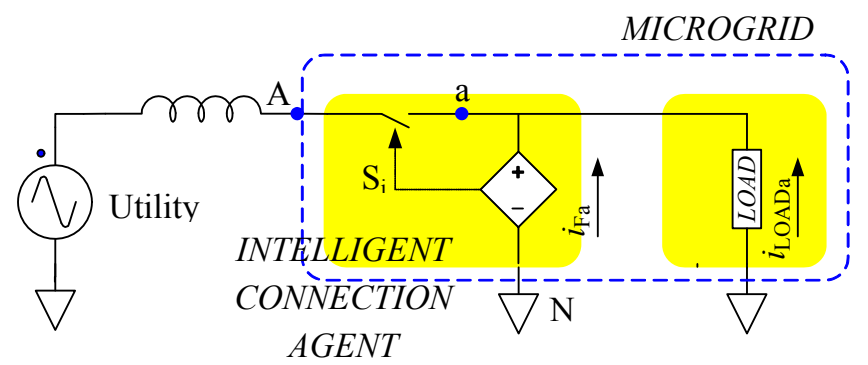

Fig. 1. Equivalent per phase network representation of the microgrid analised governed with the microgrid management agent. stand-alone or a interconnected grid, as shown in [6].

\section{B. Islanding Mode}

When a grid fault occurs, and it is detected, the VSI changes to work in islanding mode and the switch $S_{i}$, in Fig. 1, remains opened. The microgrid VSI comes to act as a grid-forming and the energy is provided from an external dc source, that could be either a battery or another dc source. In this mode, the microgrid could be composed by other sources, for example, a current source inverter supplied PV pannels. In this case, the VSI supplies or stores the necessary amount of energy to maintain the microgrid energy balance and keep frequency and voltage within the tolerance limits.

In the island mode, the control of the VSI is designed to act as a grid forming element, with the objective of setting the grid voltage amplitude, frequency and phase. The main difference in the control scheme between the island and grid connected modes lies on the fact that in the island mode there is not any ac voltage available for starting the VSI voltage reference. Thus, the VSI himself generates the ac voltage of the microgrid. This voltage is sensed and supplied to the VSI controller as the main feedback signal. In the control scheme presented in this paper, a "sample and hold" block is used to retain the grid voltage just before the grid fault is detected and the island operation mode is triggered. This 'frozen' voltage will set the initial setpoint of the grid forming VSI when operating in island mode.

\section{Transient Operating Mode}

Under deficient grid voltage conditions, the main switch $\left(S_{i}\right)$ is turned off and disconnects the microgrid from the utility. This transient should be as fast as possible in order to ensure that the load on the microgrid do not experiment any significant transient while the power flow is maintained.

In a similar way to the connection of the islanding mode, another transient state arouses when the microgrid should reconnect to the network when operating as an island. Before reclosing the main switch $S_{i}$, the ICA should synchronize the phase-angle and the magnitude of the microgrid voltage with the voltage of the utility network. Once the microgrid is linked to the main network, the VSI of the ICA changes its operation mode to the grid-connected mode.

\section{CONTROL OF THE INTELLIGENT CONTROL AGENT}

In this section the control of the ICA will be discussed when it is acting as either a voltage follower or a voltage former. In the first case the main objective of the controller is to track the voltage of the mains, while in the second case voltage-forming is intended.

\section{A. Phase-Locked Loop (PLL)}

One of the most important aspects to besolved before connecting any generation system to the utility is to perform an accurate synchronization with the network voltage. In this work, a synchronization system based on a phase locked loop (PLL) has been implemented. The objective of this PLL is to obtain the phase angle $(\theta)$ of the three-phase ac grid voltage 


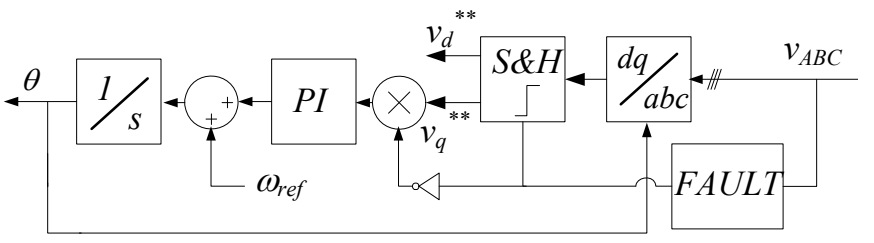

Fig. 2 Phase-lock loop basic bloc diagram used to obtain theta angle $(\theta)$ synchronized with the grid.

vector, which is expressed on a synchronous $d q$ reference frame.

The accurate estimation of this phase-angle allows both transforming the feedback variables into a synchronous reference frame, very suitable for control purposes of ac systems, and calculating and controlling the active/reactive power flow.

The PLL used in this paper has been extracted from [7], [8] and [9], and a slightly modified version it is shown in Fig.2. As it can be deduced from this figure, the three-phase grid voltage is transformed using the Clark transformation to the $\alpha-\beta$ reference frame and then the Park transformation is applied. This last transformation translates the $\alpha-\beta$ variables ac to a synchronous $d q$ reference frame. This last step permits using conventional dc integrators to detect the phase-angle of the input voltage vector. As shown in Fig. 2, the quadrature component resulting from this synchronous transformation $\left(v_{q}\right)$ is conducted to zero by using a PI controller. The output of this PI controller, added to a feedforward term representing the rated value of the grid frequency, is the rotating frequency of the synchronous reference frame. Integration of this frequency results in the phase-angle of the synchronous reference frame $(\theta)$. When the PLL is perfectly synchronized, $v_{q}$ is equal to zero, which means that the phase-angle of the synchronous reference frame matches the phase-angle of the input voltage vector.

The dynamic of the PI controller determines the performance of the PLL. Therefore, the bandwidth of the system is a tradeoff between the PI filtering performance and the time response. So, it is important remember that this parameters of the loop, $k_{P}$ and $k_{I}$ constants of the PI controller has an influence both on the detection quality of the $\theta$ phaseangle and on the PLL dynamics. In this paper, slow PLL dynamics has been choosed to attenuate the effect of the voltage harmonics on the grid synchronization process. However, if the synchronization algorithm would be used to detect grid faults, the system dynamics had to be increased

\section{B. Current and Voltage Control Loops}

The current and voltage control diagram of the IAC is shown in Fig.3. As it can be deduced from the figure, fthe voltage and the current control loops have been implemented using PI controllers working on the $d-q$ synchronous reference frame. An inner current control loop is formed around the filter inductor, and it is designed to have a very fast response. Moreover, an outer voltage control loop is in charge of tracking the grid voltage. The dynamics of this outer voltage loop is slower than the one for the current loop. This grid-follower control works as an active observer of the main grid and it is also responsible of maintaining the voltage reference when the ac bus of the microgrid is disconnected from the utility.

\section{Virtual Impedance Method}

The impedance has a considerable effect on the power sharing accuracy the active and reactive droop method when two or more grid-feeding converters are connected in parallel. The line impedance is virtually modified with the virtual

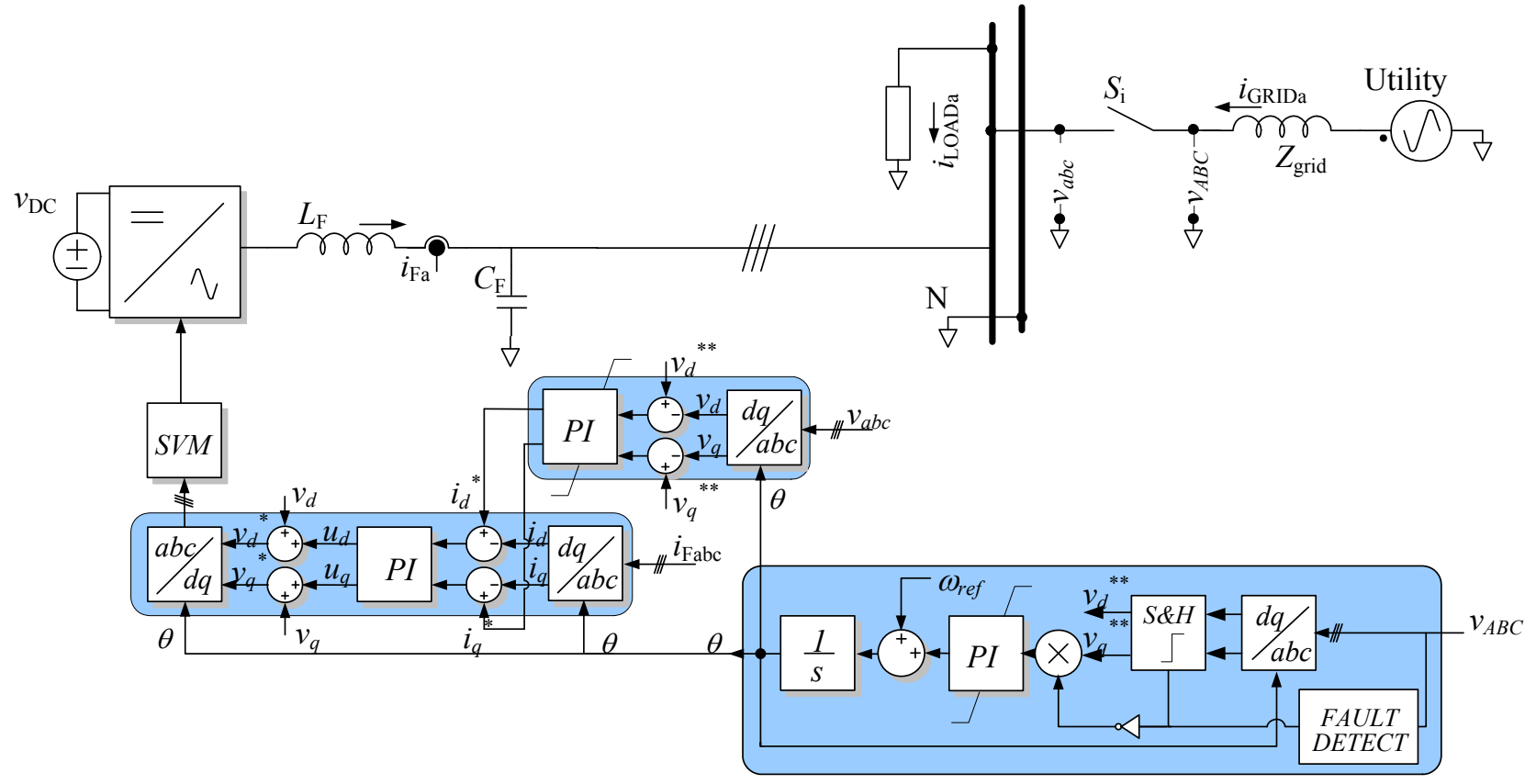

Fig. 3. Overall control blocks implementation of the proposed control diagram. This scheme is implemented and simulated through co-simulation with MATLAB and PSIM. 
impedance method, it is implemented in [10] with a resistive virtual droop method. This impedance should be larger than the real output impedance. Otherwise, any modification on the virtual output impedance will not create any effect on the power injected into the network.

As shown in (1), the initial voltage reference $\left(v^{*}{ }_{r e f}\right)$ is modified by creating a virtual voltage drop across the virtual impedance $Z_{V}$ when flowing a given output current $i_{\text {grid. }}$. The resulting voltage reference $v_{r e f} * *=\left\{v_{d}^{* *}, v_{q}^{* *}\right\}$ is provided as the input to the outer voltage control loop, [11].

$$
v_{\text {ref }}=v_{\text {ref }}^{*}-Z_{V} \cdot i_{\text {grid }} \text {. }
$$

The virtual impedance, $Z_{V}$, can be considered as a control variable and its value must be set in concordance with the nominal power of each inverter. This modification of voltage reference as a function of output current and the output has an electric meaning. This is shown in Fig. 4 and its implementation in the control loop using a PI voltage controller is shown in Fig. 3. In this system, the virtual impedance is implemented in the PI controller with future aim to increase the number of grid-feeding inverters.

It is also very important mention that no losses are actually associated to this impedance due its virtual nature, so it is possible imitate the behavior of the resistance without compromising the efficiency of the system overall.

\section{Simulation RESUlts}

The performance of the proposed control has been simulated. The VSI, shown in Fig. 1 and Fig. 3, has been simulated with the complete control system. Considering the diagram of Fig. 1, the loads are considered to be passive and with a linear behavior. The simulated system is composed by the utility grid, with the line impedance and an active switch governed by the supervisor control. This switch is used to connect, or disconnect, the microgrid to the main grid. This ac bus is also responsible of feeding the loads. In this case the load is purely resistive.

In this simulation the system operates in a grid-connected mode and the load is fully fed by the grid, while the VSI only tracks the grid voltage. Then, at $t=1.005 \mathrm{~s}$, an ideal and symmetrical voltage dip of $95 \%$ is applied at the point of common coupling (PCC), simulating a fault in the utility distribution grid.

The model has been implemented in co-simulation with Simulink/MATLAB and Psim. The environment and the results

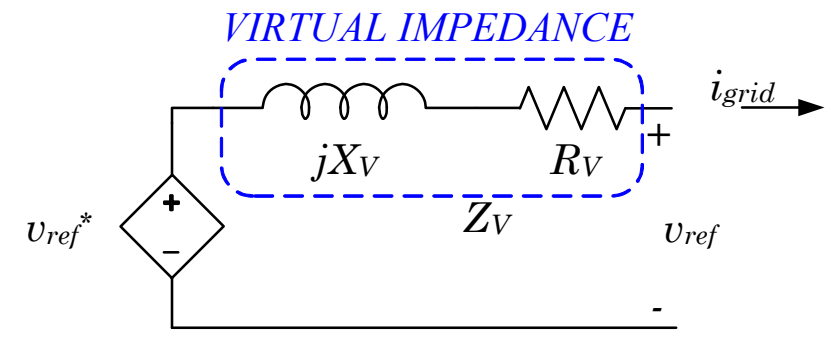

Fig. 4 Equivalent impedance representation of the virtual impedance concept.

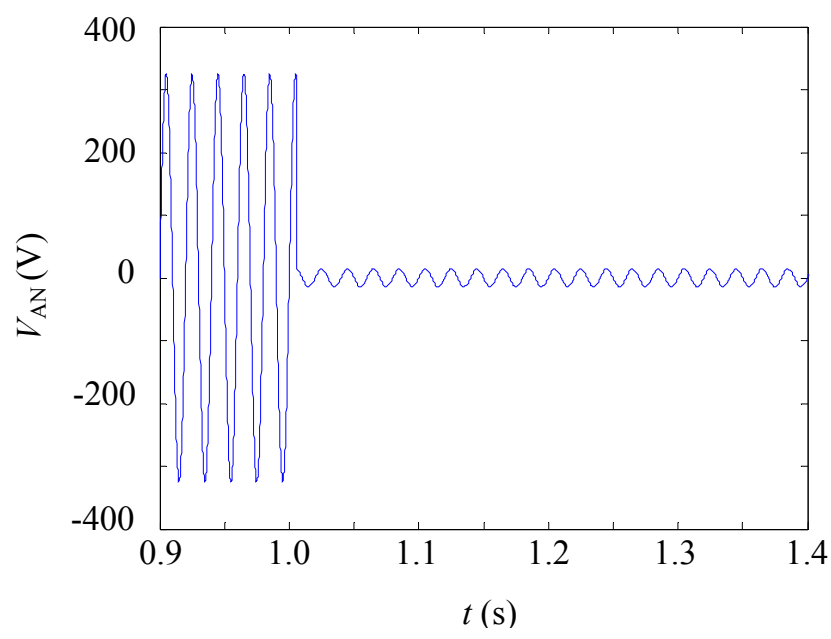

Fig. 5. Grid voltage waveform during a symmetrical grid fault applied on three voltage grid phase. The symmetrical fault occurs at $1.005 \mathrm{~s}$ and is a voltage dip of $95 \%$ of its nominal value.

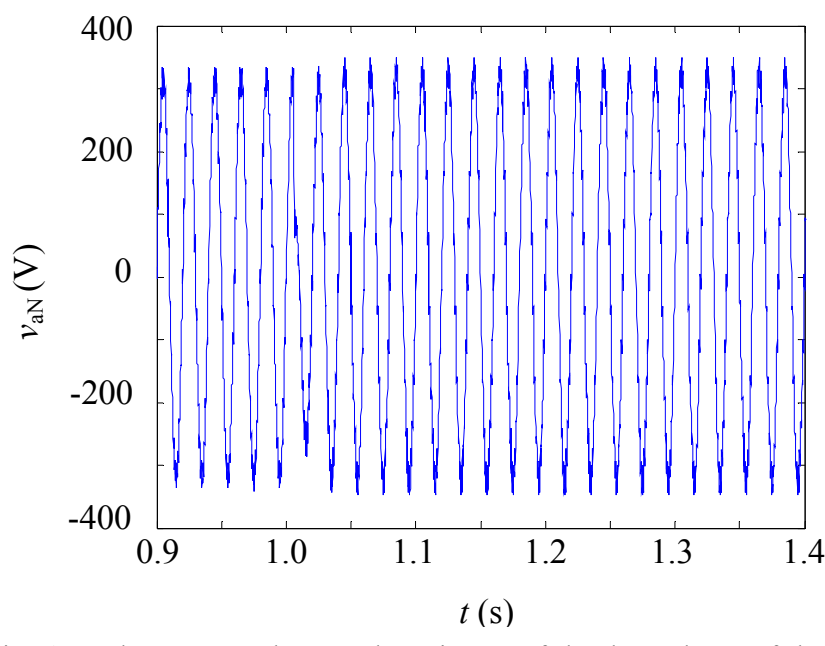

Fig. 6. Voltage across the ac voltage in one of the three phases of the microgrid under a grid fault in $1.005 \mathrm{~s}$.

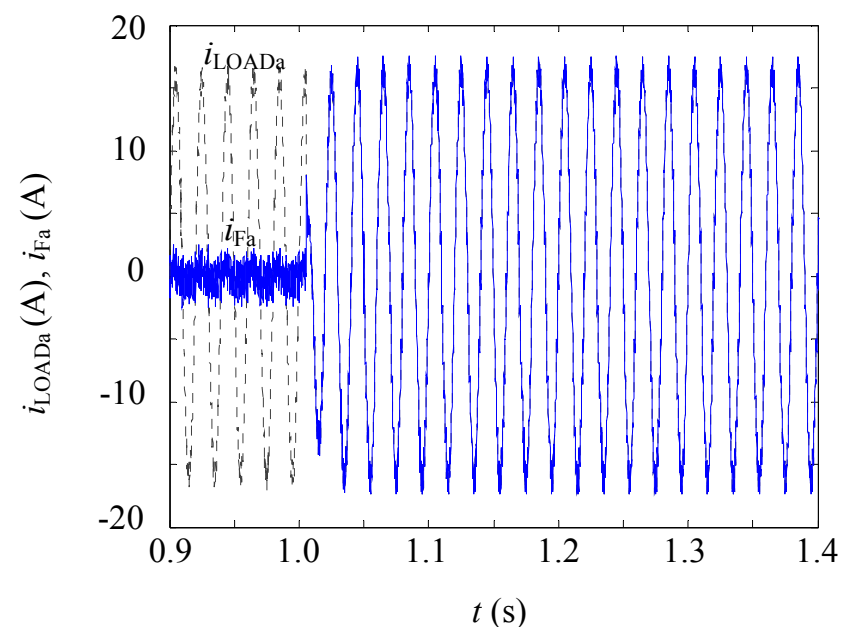

Fig. 7. Current simulation results: The dashed line represents the load current during grid-connected mode, transient and island mode. Solid line represents the current injected by the voltage source inverter due to the grid fault. The transients occurs at $\mathrm{t}=1.005 \mathrm{~s}$ 
are shown in the figures below, from Fig 5 to Fig. 8. For the sake of clarity, only one phase of the currents and voltages are shown, although all the simulations had been implemented in three phase systems. The simulation parameters used are given in Table I.

TABLE I

SIMULATION PARAMETERS

\begin{tabular}{lcc}
\hline \hline \multicolumn{1}{c}{ Parameter } & Symbol & Value and Units \\
\hline Filter Inductance & $L_{F}$ & $2.47 \mathrm{mH}$ \\
Filter Capacitance & $C_{F}$ & $20 \mu \mathrm{F}$ \\
dc voltage source & $V_{D C}$ & $700 \mathrm{~V}$ \\
Grid Inductance & $L_{G R I D}$ & $1 \mathrm{mH}$ \\
Grid Resistance & $R_{G R I D}$ & $0.5 \Omega$ \\
Resistive Load & $R_{L O A D}$ & $20 \Omega$ \\
Voltage Loop PI constant values & $k_{P}, k_{I}$ & $0.02,10$ \\
Current Loop PI constant values & $k_{P}, k_{I}$ & 20,100 \\
\hline \hline
\end{tabular}

\section{A. Main Grid Disconnection}

In Fig 5 and Fig 6. a stable grid-connected mode is observed until the line failure is applied. In a voltage line of $230 \mathrm{~V}_{\mathrm{rms}}$, at PCC a suddenly reduction of $95 \%$ is done. Subsequent to the islanding event and upon its detection, the switch $S_{i}$ is opened and grid-forming control is activated. In Fig. 5 the voltage waveforms that are produced by this fault, $v_{\mathrm{AN}}$, are shown.

The implemented control system includes a voltage normative range detection system, thus when the voltage is out of range the main switch $S_{i}$ is opened and the ICA acts as a grid-forming element, where the frequency and amplitude set point is taken from the values of the voltage detected before the fault.

When a fault occurs, the voltage measurement in the PLL is cancelled and $\omega$ remains constant to $\omega_{\text {ref }}$, which is finally used to obtain $\theta$. In grid-connected mode the block "sample $\&$ hold" give at the output the same input value, but under a grid fault gives the last value acquired in the grid-connected mode. The results obtained from simulation are presented

The Fig. 6 shows the phase voltage in the ac bus of the microgrid, $v_{\mathrm{aN}}$ during the fault: It can be noticed in the figure that the ac microgrid could be maintained without a significant disturbance of the voltage at the load's windings. In Fig. 7 the current in solid line represents the current injected by the VSI during the transient and the dashed line represents the current absorbed by the load.

Finally, in Fig. 8, the three phase voltages and currents generated at the output of the voltage source inverter are represented. From this figure it can be clearly seen that while the main network was available the current injected by the dcac inverter was null. On the other hand, when there is a contingency in the network the system switch to island mode, starting hence to feed the loads at the ac bus.

As discussed above the control loops are implemented in the $d-q$ reference frame, in order to implement both PI controllers. These give us the dynamic desired with enough precision. In Fig. 9 the $d-q$ values of the sensed voltage are shown. The objective, during and after the fault, is to maintain the initial levels or, in other words, provide in the

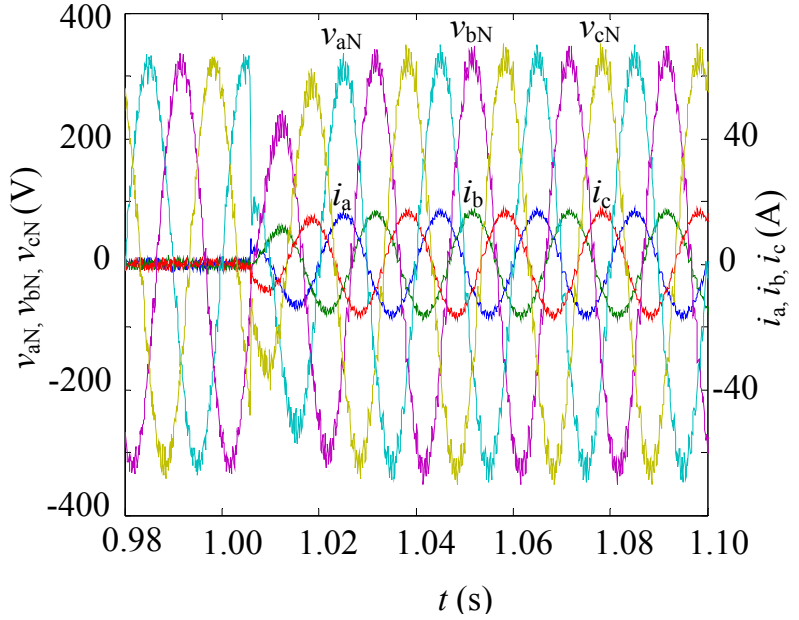

Fig. 8. Zoom in on the microgrid voltage and current circulating in the load during the microgrid disconnection of the main grid.

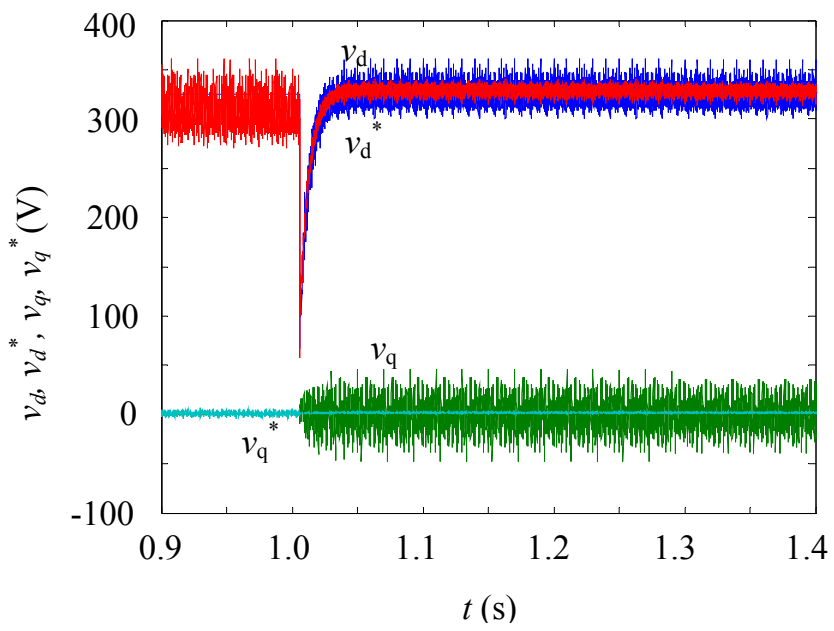

Fig. 9. Comparison between d-q voltage output references from the control blocs and d-q measured voltages during the transient from the grid-connected mode to islanding mode.

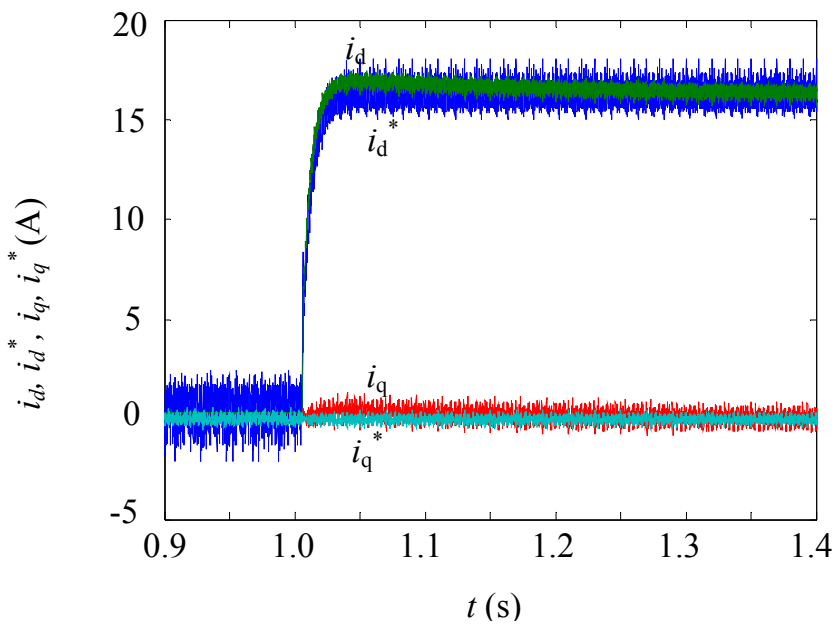

Fig. 10. Comparison between d-q current output references from the control blocks and d-q measured currents during the transient from the gridconnected mode to islanding mode. 
island mode the nominal voltage and frequency value in phase with the voltage detected before the fault. The quadrature component is zero at all times, because it works with the voltage and current in phase in all moment. The transient in the $v_{d}{ }^{*}$ is due to its generation procedure. As it can be seen in Fig. 3 the reference voltage is formed with the voltage across the $L_{F}$, function of the load current. So, a change in the load current produces a transient in the reference voltage. It can be observed that the measured values follow its reference values.

In Fig. 10 the direct and quadrature components are presented. In grid-connected mode both values are zero. In this mode all the power will be injected by the main grid. When the fault is detected, the transient starts. The steadystage is achieved again in two cycles. In island mode, with no other source of energy, all power will be injected by the voltage source inverter. This will be regulated in order to maintain the ac bus voltage among the established levels. These figures show a good performance and a fast dynamic response of the ICA. As it can be concluded from Fig. 9 as in Fig. 10 a low-pass filter can be added to remove switching noise.

\section{CONCLUSION}

This paper proposes a control strategy of a voltage source inverter to operate as a microgrid intelligent connection agent to form a secure ac bus. The proposed agent has been programmed to control the disconnection or reconnection to the main electrical grid, giving rise to excellent results.

The simulations results, obtained under co-simulation with MATLAB/simulink and Psim, had shown the good performance and a fast dynamics of the proposed system. Also, in grid-connected mode the proposed controller is capable to follow the voltage grid, in amplitude, frequency and phase. In islanding mode, the ICA presents a good regulation feeding the loads with a specific voltage, frequency and phase.

In the transition from a grid-connected mode to an islanded one, a good regulation and a fast dynamic is observed to maintain the setup voltage levels.

\section{ACKNOWLEDGMENT}

This work was supported by the Ministerio de Ciencia y Tecnología, Madrid, Spain, under Grant ENE2008-06841C02-01/ALT.

\section{REFERENCES}

[1] Z. Ye, A. Kolwalkar, Y. Zhang, P. Du, and R. Walling, "Evaluation of anti-islanding schemes based on nondetection zone concept," Power Electronics, IEEE Transactions on, vol. 19, pp. 1171-1176, 2004.

[2] M. Liserre, A. Pigazo, A. Dell'Aquila, and V. M. Moreno, "An AntiIslanding Method for Single-Phase Inverters Based on a Grid Voltage Sensorless Control," Industrial Electronics, IEEE Transactions on, vol. 53, pp. 1418-1426, 2006.

[3] J. Matevosyan, T. Ackerman, S. Bolik, and S. Lennart, "Comparison of international regulations for connection of wind turbines to the network," presented at Nordic wind power conference, 1-2 March 2004.
[4] H. Karimi, A. Yazdani, and R. Iravani, "Negative-Sequence Current Injection for Fast Islanding Detection of a Distributed Resource Unit," Power Electronics, IEEE Transactions on, vol. 23, pp. 298-307, 2008.

[5] J. M. Guerrero, N. Berbel, J. Matas, J. L. Sosa, and L. G. de Vicuna, "Control of Line-Interactive UPS Connected in Parallel Forming a Microgrid," presented at Industrial Electronics, 2007. ISIE 2007. IEEE International Symposium on, 2007.

[6] D. Georgakis, S. Papathanassiou, N. Hatziargyriou, A. Engler, and C. Hardt, "Operation of a prototype microgrid system based on microsources quipped with fast-acting power electronics interfaces," presented at Power Electronics Specialists Conference, 2004. PESC 04. 2004 IEEE 35th Annual, 2004.

[7] A. Timbus, R. Teodorescu, F. Blaabjerg, and M. Liserre, "Synchronization Methods for Three Phase Distributed Power Generation Systems. An Overview and Evaluation," presented at Power Electronics Specialists Conference, 2005. PESC '05. IEEE 36th, 2005.

[8] P. Rodriguez, "Aportaciones a los acondicionadores activos de corriente en derivación para redes trifásicas de cuatro hilos," in Electrical Engineering. Barcelona: Universitat Politècnica de Catalunya, 2005, pp. 416.

[9] V. Kaura and V. Blasko, "Operation of a phase locked loop system under distorted utility conditions," Industry Applications, IEEE Transactions on, vol. 33, pp. 58-63, 1997.

[10] S. J. Chiang, C. Y. Yen, and K. T. Chang, "A multimodule parallelable series-connected PWM voltage regulator," Industrial Electronics, IEEE Transactions on, vol. 48, pp. 506-516, 2001.

[11] J. M. Guerrero, L. Hang, and J. Uceda, "Control of Distributed Uninterruptible Power Supply Systems," Industrial Electronics, IEEE Transactions on, vol. 55, pp. 2845-2859, 2008. 